Pacific Journal of Mathematics

SEMICLOSED OPERATORS

Vol. 44, No. 1

May 1973 


\title{
SEMICLOSED OPERATORS
}

\author{
S. R. CARADUS
}

Elementary wellknown examples show that the sum of two closed operators need not even have a closed extension; the same is true for products, as one can see by taking the composition of maps $f \rightarrow f^{\prime}$ followed by $f \rightarrow f(0)$ defined on the obvious domains in $C[0,1]$. The natural question which then arises concerns the complexity of operators which might arise by taking repeated sums and products, starting with the closed operators. Somewhat unexpectedly, the answer is very simple: all can be reduced to products of two closed operators. Because of this, we shall distinguish this latter class by the name "semiclosed".

The most convenient proof of this theorem is obtained by first showing that semiclosed operators can always be decomposed in certain special ways.

Lemma 1. (Canonical Decomposition). Let $X$ and $Y$ be Banach spaces and let $T: X \rightarrow Y$ denote a semiclosed linear operator. Then there exists a Banach space $Z$ and closed operators $U: Z \rightarrow Y$ and $V: X \rightarrow Z$ with the following properties:

(a) $T=U V$

(b) $U$ is defined and continuous on all of $Z$ and the range of $U$ is exactly the range of $T$.

(c) $V$ has the same domain as $T$ and maps this domain one-toone onto $Z$.

Proof. Since $T$ is semiclosed, there exists some decomposition $T=P Q$ where $P: W \rightarrow Y$ and $Q: X \rightarrow W$ are closed linear mappings and $W$ is some Banach space. Then define

$$
Z=\{(x, Q x, P Q x): x \in D(T)\}
$$

where $D(T)$ denotes the domain of $T$ and $Z$ is considered as a subspace of $X \times W \times Y$. Next take

$$
V: x \longmapsto(x, Q x, P Q x)
$$

and

$$
U:(x, Q x, P Q x) \longmapsto P Q x
$$

with $D(V)=D(T)$ and $D(U)=Z$. Then the properties (a), (b) and (c) are easily verified. 
We shall reserve the special notation $T=U V(Z)$ to represent the decomposition described in the above lemma. It is clear that, given $T$, the space $Z$ is unique up to isomorphism. When $T$ is a bounded operator with $D(T)=X$, we have $T=T I(X)$ and when $T$ is closed, we can write $T=P G(G(T))$ where $G(T)$ denotes the graph of $T, G$ is the mapping $x \mapsto(x, T x)$ and $P$ the projection $(x, T x) \mapsto T x$. Another immediate consequence of Lemma 1 is the fact that a semiclosed operator with closed domain is continuous.

Finally, it is interesting to recall the well-known procedure of "making a closed operator continuous" by renorming it domain with the graph norm $|x|_{T}=|x|+|T x|$. Lemma 1 implies that the existence of such a procedure characterises semiclosed operators. We make this precise as follows:

Corollary 1. Let $T: X \rightarrow Y$ denote an arbitrary linear operator. Then $T$ is semiclosed if and only if there exists a norm $x \rightarrow|x|_{T}$ on $D(T)$ such that (a) the normed space $X_{T}=\left(D(T),|\cdot|_{T}\right)$ is complete (b) the induced operator $\widetilde{T}: X_{T} \rightarrow Y$ is continuous.

Proof. If $T$ is semiclosed and $T=U V(Z)$, then define $|x|_{T}=$ $|V x|$. Conversely, if $|\cdot|_{T}$ exists with properties (a) and (b), then define $T=U V\left(X_{T}\right)$ with $V x=x$ and $U x=T x$.

THEOREM 1. If $T_{1}$ and $T_{2}$ are semiclosed operators, then so are $T_{1}+T_{2}$ and $T_{1} T_{2}$ (whenever the latter are defined).

Proof. Suppose we have $T_{i}=U_{i} V_{i}\left(Z_{i}\right), i=1,2$. Then we simply construct the required decompositions.

(i) Let $W=\left\{\left(x, V_{1} x, V_{2} x\right): x \in D\left(T_{1}+T_{2}\right)\right\} \subseteq X \times Z_{1} \times Z_{2}$; $V: X \rightarrow W, D(V)=D\left(T_{1}+T_{2}\right), \quad V x=\left(x, V_{1} x, V_{2} x\right) ; \quad D(U)=W$, $U\left(x, V_{1} x, V_{2} x\right)=\left(T_{1}+T_{2}\right) x$. Then $T_{1}+T_{2}=U V(W)$.

(ii) Let $\hat{W}=\left\{\left(x, V_{2} x, V_{1} T_{2} x\right): x \in D\left(T_{1} T_{2}\right)\right\} \leqq X \times Z_{2} \times Z_{1} ; \hat{V}: X \rightarrow$ $\hat{W}, D(\hat{V})=D\left(T_{1} T_{2}\right), \hat{V} x=\left(x, V_{2} x, V_{1} T_{2} x\right) ; D(\hat{U})=\hat{W}, \hat{U}\left(x, V_{2} x, V_{1} T_{2} x\right)=$ $T_{1} T_{2} x$. Then $T_{1} T_{2}=\hat{U} \hat{V}(\hat{W})$.

Verifications of the above assertions are straight forward.

The above theorem shows that the property of being semiclosed is algebraically stable. In addition, it persists in other useful ways.

THeOREM 2. Let $T: X \rightarrow Y$ denote a semiclosed operator. Then

(i) if $X$ is separable (or more generally, if $X$ admits quasicomplements [7], [5]) then $T$ has a densely defined semiclosed extension. 
(ii) if $X_{0}$ is a subspace of $X$ and $X_{0}$ is the domain of some closed operator, then $T_{0}$, the restriction of $T$ to $X_{0}$, is a semiclosed operator.

Proof. (i) Suppose $D(T)$ is not dense in $X$ and write $D=\overline{D(T)}$. Then if $\hat{D}$ is a quasicomplement of $D$, we can define the projection map $\pi: D \oplus \hat{D} \rightarrow D$. It is easy to verify that $\pi$ is closed so that $T \pi$ is a semiclosed extension of $T$. Finally, straight forward arguments show that $T \pi$ is densely defined. (ii) If $X_{0}$ is the domain of some closed operator, then $X_{0}$ is also the range of a closed operator $S: Z \rightarrow X$ for some $Z$. Hence $X_{0}$ is also the range of a one-to-one closed operator $\hat{S}: Z / N(S) \rightarrow X$. Now $\hat{S} \hat{S}^{-1}$ is semiclosed and is the restriction $I_{0}$ of the identity operator to $X_{0}$. Hence $T I_{0}=T_{0}$ is semiclosed.

REMARK. It is known that not every subspace is the domain of a closed operator. Kaashoek [3] draws attention to this point by giving a simple construction in Banach space of a dense subspace of finite codimension. A wellknown theorem [4] indicates that such a subspace cannot be the range of any closed operator and hence cannot be the domain of any closed operator.

We now introduce a topology in the class $\mathscr{S}(X, Y)$ of semiclosed operators $X \rightarrow Y$. Let $T \in \mathscr{S}(X, Y)$ and suppose $\alpha$ denotes a canonical decomposition $T=U V(Z)$ for $T$. Then, for $\varepsilon>0$, write

$$
\begin{aligned}
\mathscr{N}(T ; \alpha, \varepsilon)= & \{S \in \mathscr{S}(X, Y): D(S)=D(T), \\
& S \text { has canonical decomposition } S=\widetilde{U} V(Z), \\
& \|U-\widetilde{U}\|<\varepsilon\} .
\end{aligned}
$$

The topology generated in $\mathscr{S}(X, Y)$ by the semibasic sets $\mathscr{N}(T ; \alpha, \varepsilon)$ will be denoted by $\tau$. Since applications visualized and examples in the current literature (e.g. [8]) involve families of operators which are all defined on the same domain, the above definition is not as restrictive as would appear. It is also possible to show that it suffices to choose, for each $T$, just one canonical decomposition $\alpha_{T}$; the topology generated is the same.

The next thing to observe is that, if $B(X, Y)$ denotes the space of bounded operators defined on all of $X$, then $\tau$ restricted to $B(X, Y)$ is the uniform operator topology. For, on the one hand, it is evident that $\{S \in B(X, Y):\|S-T\|<\varepsilon\} \subseteq \mathscr{N}(T ; \alpha, \varepsilon)$ where $T \in B(X, Y)$ and $\alpha$ is given by $T=T I(X)$. Conversely if $\beta$ is any canonical decomposition for $T \in B(X, Y)$ then, by uniqueness, $\beta$ has the form $T \phi . \phi^{-1}(Z)$ where $\phi$ is an isomorphism of $Z$ onto $X$. Hence if $S \in \mathscr{N}(T ; \beta, \varepsilon) \cap B(X, Y)$, then $S$ can be written $S \dot{\phi} \cdot \phi^{-1}(Z)$ and 
thus $\mathscr{N}(T ; \beta, \varepsilon) \cap B(X, Y) \subseteq\left\{S \in B(X, Y):\|S-T\|<\varepsilon\left\|\phi^{-1}\right\|\right\}$.

The relationship between $\tau$ and class of closed operators in $\mathscr{S}(X, Y)$ is not so evident. A well known "generalised convergence" for closed operators is throughly treated in [4]. An application of Theorem IV. 2.29 of [4] shows that if $\left\{T_{n}\right\}$ is a sequence of closed operators converging to closed operator $T$ in the topology $\tau$, then $\left\{T_{n}\right\}$ also converges to $T$ in the generalised sense.

Returning now to the study of $\tau$ on $\mathscr{S}(X, Y)$, we can show that $\tau$ has all reasonable properties for which one might hope.

THEOREM 3. $\tau$ is a locally convex Hausdorff topology on $\mathscr{S}(X, Y)$.

Proof. We will show, in fact, that each $\mathscr{N}(T ; \alpha, \varepsilon)$ is convex. Let $S_{i} \in \mathscr{N}(T ; \alpha, \varepsilon), i=1,2$ with $\alpha$ given by $T=U V(Z)$. Then $S_{i}$ can be written $U_{i} V(Z)$ with $\left\|U-U_{i}\right\|<\varepsilon$. If $a_{i}>0$ with $a_{1}+a_{2}=1$, then $a_{1} S_{1}+a_{2} S_{2}=\bar{U} V(Z)$ where $\bar{U}(V x)=a_{1} S_{1}+a_{2} S_{2} x$. Hence

$$
\|(U-\widetilde{U}) V x\|=\left\|a_{1}\left(U-U_{1}\right) V x+a_{2}\left(U-U_{2}\right) V x\right\| \leqq \varepsilon\|V x\| \cdot
$$

Secondly, suppose $T_{1}$ and $T_{2}$ are semiclosed operators not separated by $\tau$. Then in particular, for each $\varepsilon>0$ and canonical decompositions $\alpha_{1}$ and $\alpha_{2}$, respectively, for $T_{1}$ and $T_{2}$, there must exist $S \in \mathscr{N}\left(T_{1} ; \alpha_{1}, \varepsilon\right) \cap \mathscr{N}\left(T_{2} ; \alpha_{2}, \varepsilon\right)$. Evidently $D\left(T_{1}\right)=D\left(T_{2}\right)$ and without loss of generality, we may restrict considerations to $\alpha_{1}$ and $\alpha_{2}$ acting through the same intermediate space $Z$. So we have $T_{i}=$ $U_{i} V_{i}(Z)$ and $S$ has two decompositions $S=\widetilde{U}_{i} V_{i}(Z)$ such that \| $\widetilde{U}_{i}-U_{i} \|<\varepsilon, i=1,2$. By uniqueness, there exists an automorphism $\phi$ of $Z$ such that $V_{1}=\phi V_{2}$ and $\widetilde{U}_{2}=\widetilde{U}_{1} \phi$. Hence, for any $x \in D\left(T_{1}\right)=$ $D\left(T_{2}\right)$, we have

$$
\begin{aligned}
\left\|\left(T_{1}-T_{2}\right) x\right\| & =\left\|\left(U_{1}-\widetilde{U}_{1}\right) V_{1} x+\left(\widetilde{U}_{2}-U_{2}\right) V_{2} x\right\| \\
& \leqq \varepsilon\left(\left\|V_{1} x\right\|+\left\|V_{2} x\right\|\right) .
\end{aligned}
$$

By holding the $\alpha_{i}$ fixed, we see that, for each $x, T_{1} x-T_{2} x$ can be made arbitrarily small. Hence $T_{1}=T_{2}$.

THEOREM 4. The mappings $\left(T_{1}, T_{2}\right) \rightarrow T_{1}+T_{2}$ and $(\lambda, T) \rightarrow \lambda T$ are jointly continuous in $\mathscr{S}(X, Y)$. Moreover if $X=Y$, then $\left(T_{1}, T_{2}\right) \rightarrow T_{1} T_{2}$ is separately continuous.

Proof. (Sketch) Using by now familiar methods, we can obtain relations such as

$$
\mathscr{N}\left(T_{1} ; \alpha_{1}, \varepsilon_{1}\right)+\mathscr{N}\left(T_{2} ; \alpha_{2}, \varepsilon_{2}\right) \subseteq \mathscr{N}\left(T_{1}+T_{2} ; \alpha_{1}+\alpha_{2}, \varepsilon_{1}+\varepsilon_{2}\right)
$$


where $\alpha_{1}+\alpha_{2}$ is obtained from $\alpha_{1}$ and $\alpha_{2}$ by the construction of Theorem 1. Similar relations for multiplication are obtained.

We now turn attention toward possible applications. Our ultimate goal would be to subsume portions of the theory of partial differential operators with constant coefficients in a sufficiently general understanding of the topological algebras generated by the closed operators which can be obtained from the differentiation operations. We would therefore be considering commutative topological algebras obtained by fixing $n$ commuting generators $D_{1} \cdots D_{n}$ in $\mathscr{S}(X, X)$, all defined on the same domain. The development of the theory of such algebras has almost exclusively involved additional assumptions which have the effect of making the spectrum of each element in the algebra a compact subset of the complex plane. See for example [1] and [6]. In our case, however, we must allow unbounded spectra if we are to obtain information about spectral properties of differential operators. While it is true that some progress in this direction has been made in [9] and [10], much work is yet required before the applications envisaged above can be carried through.

\section{REFERENCES}

1. A. Guichardet, Special Topics in Topological Algebras, Gordon and Breach 1968.

2. E. Hille and R. S. Phillips, Functional Analysis and Semigroups, Revised Edition, American Math. Soc., 1957.

3. M. A. Kaashoek, Closed linear operators on Banach spaces, Thesis, University of Leiden, 1964.

4. T. Kato, Perturbation Theory for Linear Operators, Springer-Verlag, 1966.

5. J. Lindenstrauss, An. Acad. Brasil. Ci. 39 (1967), 1-6 Israel J. Math. 6 (1968), 36-38.

6. E. A. Michael, Locally Multiplicatively-Convex Topological Algebras, Memoris Amer. Math. Soc., 11, 1952.

7. F. J. Murray, Trans. Amer. Math. Soc., 58 (1945), 77-95.

8. R. T. Powers, Self Adjoint Algebras of Unbounded Operators, Comm. Math. Phys., 21 (2) (1971), 85-124.

9. J. Sebastiao e Silva, Sur le calcul symbolique d' operateurs permutables, a spectre vide ou non borne, Ann. Mat. Pura. Appl., (4) 58 (1962) 219-275.

10. L. Waelbroeck, Etudes spectral de certaines algebres completes, Colloque sur L'Analyse Fonctionnelle, CBRM (1961), 29-38.

11. G. W. Mackey, Bull. Amer. Math. Soc., 52 (1946), 322-325.

Received September 8, 1971. Research partially supported by NRC Operating Grant A3985.

QUEEN'S UNIVERSITY

KINGSTON, ONTARIO 



\section{PACIFIC JOURNAL OF MATHEMATICS}

\section{EDITORS}

H. SAMELSON

Stanford University

Stanford, California 94305

C. R. Hовву

University of Washington

Seattle, Washington 98105
J. DugundJI

Department of Mathematics University of Southern California

Los Angeles, California 90007

RICHARD ARENS

University of California

Los Angeles, California 90024

\section{ASSOCIATE EDITORS}

E. F. BECKENBACH

B. H. NeumanN

F. WOLF

K. YoSHIDA

\section{SUPPORTING INSTITUTIONS}

UNIVERSITY OF BRITISH COLUMBIA

CALIFORNIA INSTITUTE OF TECHNOLOGY

UNIVERSITY OF CALIFORNIA

MONTANA STATE UNIVERSITY

UNIVERSITY OF NEVADA

NEW MEXICO STATE UNIVERSITY

OREGON STATE UNIVERSITY

UNIVERSITY OF OREGON

OSAKA UNIVERSITY
UNIVERSITY OF SOUTHERN CALIFORNIA

STANFORD UNIVERSITY

UNIVERSITY OF TOKYO

UNIVERSITY OF UTAH

WASHINGTON STATE UNIVERSITY

UNIVERSITY OF WASHINGTON

AMERICAN MATHEMATICAL SOCIETY

NAVAL WEAPONS CENTER

Printed in Japan by International Academic Printing Co., Ltd., Tokyo, Japan 


\section{Pacific Journal of Mathematics}

\section{Vol. 44, No. $1 \quad$ May, 1973}

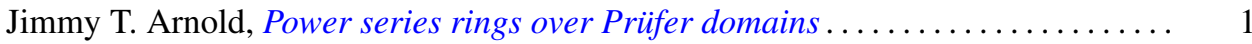

Maynard G. Arsove, On the behavior of Pincherle basis functions . . . . . . . . . 13

Jan William Auer, Fiber integration in smooth bundles ................. 33

George Bachman, Edward Beckenstein and Lawrence Narici, Function algebras

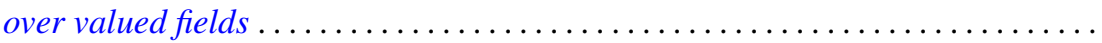

Gerald A. Beer, The index of convexity and the visibility function . . . . . . . . . . .

James Robert Boone, A note on mesocompact and sequentially mesocompact

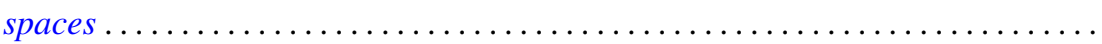

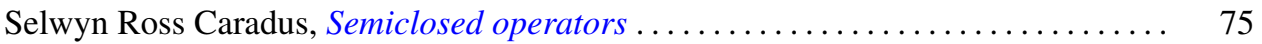

John H. E. Cohn, Two primary factor inequalities . . . . . . . . . . . . . . . 81

Mani Gagrat and Somashekhar Amrith Naimpally, Proximity approach to

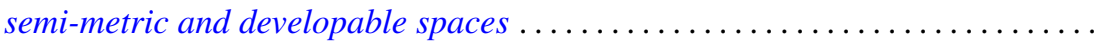

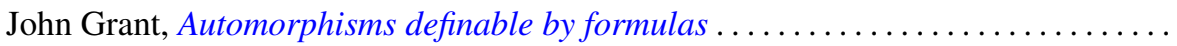

Walter Kurt Hayman, Differential inequalities and local valency ..............

Wolfgang H. Heil, Testing 3-manifolds for projective planes . . . . . . . . . . . . .

107

Melvin Hochster and Louis Jackson Ratliff, Jr., Five theorems on Macaulay

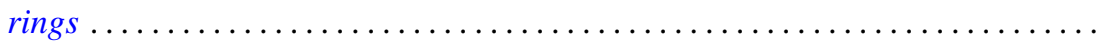

Thomas Benton Hoover, Operator algebras with reducing invariant subspaces ....

James Edgar Keesling, Topological groups whose underlying spaces are separable

Fréchet manifolds...

Frank Leroy Knowles, Idempotents in the boundary of a Lie group . .

191

George Edward Lang, The evaluation map and EHP sequences ...

201

Everette Lee May, Jr, Localizing the spectrum . . . . . . . . . . . .

211

Frank Belsley Miles, Existence of special $K$-sets in certain locally compact abelian groups.

Susan Montgomery, A generalization of a theorem of Jacobson. II . .

T. S. Motzkin and J. L. Walsh, Equilibrium of inverse-distance forces in

three-dimensions.

Arunava Mukherjea and Nicolas A. Tserpes, Invariant measures and the converse

of Haar's theorem on semitopological semigroups .

James Waring Noonan, On close-to-convex functions of order $\beta$

Donald Steven Passman, The Jacobian of a growth transformation

Dean Blackburn Priest, A mean Stieltjes type integral ........ .

Joe Bill Rhodes, Decomposition of semilattices with applications to topological

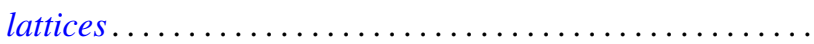

Claus M. Ringel, Socle conditions for $\mathrm{QF}-1$ rings ..........

Richard Rochberg, Linear maps of the disk algebra

Roy W. Ryden, Groups of arithmetic functions under Dirichlet convolution . .

Michael J. Sharpe, A class of operators on excessive functions

Erling Stormer, Automorphisms and equivalence in von Neumann algebras ..

Philip C. Tonne, Matrix representations for linear transformations on series

analytic in the unit disc. 